\title{
Manejo de lesão extensa face decorrente de mordedura canina em paciente pediátrico: Relato de caso clínico
}

Management of extensive facial injury resulting from canine bite in a pediatric patient: A clinical case report

Manejo de la lesión facial extensa resultante de la mordedura de un canino en un paciente pediátrico: Reporte de un caso clínico

Chiara Cristina Diógenes

ORCID: https://orcid.org/0000-0001-6180-9549 Universidade Estadual da Paraíba, Brasil E-mail: chiaracristinadgns@gmail.com

Fábio Henrique Vasques Bezerra ORCID: https://orcid.org/0000-0002-2978-8722

Universidade Estadual da Paraíba, Brasil E-mail: fabiohenriquevb@hotmail.com

Maxsuel Bezerra da Silva ORCID: https://orcid.org/0000-0003-4366-9508

Universidade de Pernambuco, Brasil

E-mail: maxsuelmd@ @otmail.com

Ingrid Torres de Almeida

ORCID: https://orcid.org/0000-0002-4661-1849

Universidade de Pernambuco, Brasil

E-mail: ingridtalmeida@gmail.com

Lucas Emmanuell de Morais Neves ORCID: https://orcid.org/0000-0001-7257-3148

Universidade de Pernambuco, Brasil

E-mail: lucas_emmanuell@hotmail.com

Frank Gigianne Teixeira e Silva ORCID: https://orcid.org/0000-0001-5115-8586 Faculdade Santa Maria, Brasil

E-mail: frankodonto@gmail.com

Amanda Galvão Souza

ORCID: https://orcid.org/0000-0002-1076-1394 Universidade de Pernambuco, Brasil E-mail: amandagalvaos@outlook.com

Ana Beatriz Leme de Andrade ORCID: https://orcid.org/0000-0003-0357-8417

Universidade de Pernambuco, Brasil

E-mail: anabeatrizleme@ hotmail.com

Fernando Antônio Cardoso Maciel

ORCID: https://orcid.org/0000-0001-8392-1718 Hospital da Restauração Governador Paulo Guerra, Brasil E-mail: fernanciel@hotmail.com

\begin{abstract}
Resumo
Os traumas na infância e adolescência constituem um fator importante de morbidade e mortalidade, sendo um enorme problema de saúde pública. Dentre os traumas faciais em crianças, as mordeduras de animais destacam-se, principalmente a canina. E este tipo de ferimento resulta em traumatismos faciais complexos, podendo ter um envolvimento funcional e estético, assim como sucedendo em infecções polimicrobianas. Paciente do sexo masculino, 8 anos de idade, compareceu ao Hospital da Restauração em Recife/Pernambuco, vítima de mordedura canina cursando com lesão lácero-contusa extensa em região de comissura labial do lado esquerdo. O tratamento instituído neste primeiro momento foi por meio de sutura em todo o ferimento e encaminhamento para vacinação anti-rábica após três dias. Foram dadas as orientações pós-operatórias de realização de curativos diários, retorno em 10 dias para remoção das suturas, retorno para acompanhamento da vacinação anti-rábica, repouso por 10 dias e orientação de higiene oral. O trauma promovido pela mordedura canina é definido como um problema de saúde público e que afeta em sua maioria a população pediátrica. Dessa forma faz-se necessário estabelecer a devida atenção ao tratamento e
\end{abstract}


acompanhamento desses pacientes, seguindo os protocolos de atendimento necessários, promovendo também, o controle de potenciais complicações que provém de lesões dessa natureza.

Palavras-chave: Ferimentos e Lesões; Face; Procedimentos Cirúrgicos Reconstrutivos.

\begin{abstract}
Trauma in childhood and adolescence is an important factor in morbidity and mortality, being a huge public health problem. Among the facial traumas in children, animal bites stand out, mainly canine. And this type of injury results in complex facial trauma, which can have a functional and aesthetic involvement, as well as occurring in polymicrobial infections. Male patient, 8 years old, attended the Hospital da Restauração in Recife / Pernambuco, victim of a canine bite with extensive blunt lesion in the region of the labial commissure on the left side. The treatment instituted in this first moment was by suturing the entire wound and referring for rabies vaccination after three days. Post-operative guidelines were given for daily dressings, return in 10 days for suture removal, return for monitoring of rabies vaccination, rest for 10 days and oral hygiene guidance. The trauma caused by canine bite is defined as a public health problem that mostly affects the pediatric population. Thus, it is necessary to establish due attention to the treatment and follow-up of these patients, following the necessary care protocols, also promoting the control of potential complications that arise from injuries of this nature.
\end{abstract}

Keywords: Wounds na Injuries; Face; Reconstructive Surgical Procedures.

\title{
Resumen
}

El trauma en la niñez y la adolescencia es un factor importante de morbilidad y mortalidad, siendo un gran problema de salud pública. Entre los traumatismos faciales en los niños destacan las mordeduras de animales, principalmente caninos. Y este tipo de lesión resulta en traumatismos faciales complejos, que pueden tener una afectación funcional y estética, además de ocurrir en infecciones polimicrobianas. Paciente masculino de 8 años que acudió al Hospital da Restauração de Recife / Pernambuco, víctima de una mordida canina con lesión contusa extensa en la comisura labial del lado izquierdo. El tratamiento instituido en este primer momento fue suturar toda la herida y derivar a vacunación antirrábica a los tres días. Se dieron pautas postoperatorias para apósitos diarios, regreso en 10 días para remoción de suturas, regreso para monitoreo de vacunación antirrábica, reposo por 10 días y guía de higiene bucal. El trauma causado por la mordedura canina se define como un problema de salud pública que afecta mayoritariamente a la población pediátrica. Por ello, es necesario establecer la debida atención al tratamiento y seguimiento de estos pacientes, siguiendo los protocolos de atención necesarios, promoviendo también el control de las potenciales complicaciones que se derivan de lesiones de esta naturaleza.

Palabras clave: Heridas y Traumatismos; Cara; Procedimientos Quirúrgicos Reconstructivos.

\section{Introdução}

As lesões em tecidos moles faciais possuem um lugar de relevância no atendimento a pacientes politraumatizados nas emergências, pois estes ferimentos podem prejudicar, permanentemente, o paciente. Quando tratado de forma inadequada, esse tipo de lesão pode vir a deixar sequelas, distanciando assim a pessoa do convívio social e até mesmo incapacitando de trabalhar, ocasionando um segregamento econômico (Vieira, Araújo, Ribeiro \& Filho, 2013; Foster \& Hudson, 2015; Macedo, Rosa, Queiroz \& Gomes, 2016; Senturk, Dagistanli, Calim \& Ozturan, 2019).

Os traumas na infância e adolescência constituem um fator importante de morbidade e mortalidade, sendo um enorme problema de saúde pública. Como principais fatores, as mordeduras de animais destacam-se, principalmente as caninas, tornando-se motivo de grande atenção na saúde pública devido a probabilidade de transmissão de raiva, doença quase sempre fatal (Carvalho \& Silva, 2007; Essig, Sheehan, Rikhi, Elmaraghy \& Christophel, 2018; Cohen-Manheim, Siman-Tov, Radomislensky, Group \& Peleg, 2018; Cavalcanti, Porto, Santos, Cavalcanti \& Cavalcanti, 2017)

Esse tipo de trauma está presente entre os 15 principais tipos de injurias não fatais na população geral abaixo dos 10 anos de idade (Zhu, Walma, Troulis \& August, 2020). Em grande maioria, os ataques são promovidos por animais domésticos e sem a presença de um adulto durante o momento do ataque (Macedo et al., 2016). Entre as raças que mais promovem ataques dessa natureza, destacam-se: Pitbull, Labrador, Pastor Alemão, Bull Terrier e Boxer (Zhu et al., 2020).

Esse tipo de ferimento resulta em traumatismos faciais complexos, podendo ter um envolvimento funcional e estético, assim como sucedendo em infecções polimicrobianas, tornando-se um desafio para os cirurgiões maxilofaciais (Piccart et al., 2018; Cohen-Manheim et al., 2018). Dada a altura de uma criança pequena e a cabeça maior em tamanho, assim como a falta 
de força dificuldade de força para defesa a prevalência de lesões por mordedura canina vai variar inversamente com a idade (Heitz, Louzada, Couci, Rodrigues \& Fritscher, 2018; Saadi, Oberman \& Lighthall, 2018; Foster \& Hudson, 2015).

A forma como o ataque de um cachorro ocorre é única, produzindo então, considerações valiosas na história inicial do trauma, reconstrução cirúrgica e profilaxia. Estes traumatismos em tecidos moles são comumente categorizados em três tipos preponderantes: laceração, avulsão e punção. A cinemática é tão intensa e penetrante que pode resultar em enormes avulsões da pele, desvitalizando tecidos moles e causando danos às estruturas neurovasculares, no entanto, as fraturas ósseas associadas são raras. Mesmo não envolvendo tecido ósseo, o tratamento é deveras complexo, sendo necessário um reparo primário dos ferimentos complexos para que se tenha um resultado o mais satisfatório possível (Heitz et al., 2018; Saadi et al., 2018; Cavalcanti et al., 2017).

$\mathrm{O}$ tratamento complementar com antibióticos é indicado para mordeduras que já possuem risco de infecção ou com evidência de infecção, de alta complexidade e quando comprometem estruturas essenciais como ossos, vasos e articulações. Deve-se considerar também a imunização contra o tétano e a possibilidade de infecção por raiva (Heitz et al., 2018; CohenManheim et al., 2018; Klerk, Dijk \& As, 2016).

A despeito de haver diversas pesquisas envolvendo o tratamento ideal para este tipo de lesão, não há evidências sólidas para que um único manejo seja padronizado. As lesões por mordedura canina são de alta complexidade e bastante graves, possuindo também experiências clínicas variadas dos cirurgiões, então, a soma destes fatores leva a falta de consenso sobre qual o tratamento ideal para as vítimas acometidas por esta fatalidade (Drumright, Borg, Rozzelle, Donoghue \& Shanti, 2020).

\section{Metodologia}

O presente ensaio é um estudo intervencional descritivo e qualitativo de relato de caso o qual possui a autorização do paciente por meio do Termo de Consentimento Livre e Esclarecido (TCLE), elaborado em linguagem acessível à compreensão do paciente relatado, evidenciando o destino das informações coletadas do paciente, assim como os benefícios e riscos associados à sua participação que foi assinado no momento de entrada no Hospital. Também foi realizada uma revisão da literatura por meio de busca de artigos científicos, priorizando-se aqueles dos últimos 10 anos e possuíssem informações relevantes e parecidas com o deste artigo, e foram utilizadas as bases de dados PubMed, Scielo e Lilacs para pesquisa.

\section{Relato de Caso}

Paciente do sexo masculino, 8 anos de idade, compareceu ao Hospital da Restauração em Recife/Pernambuco, vítima de mordedura canina cursando com lesão lácero-contusa extensa em região de comissura labial do lado esquerdo. Demonstrada na Figura 1. 
Figura 1: Fotografia de face inicial demonstrando ferimento.

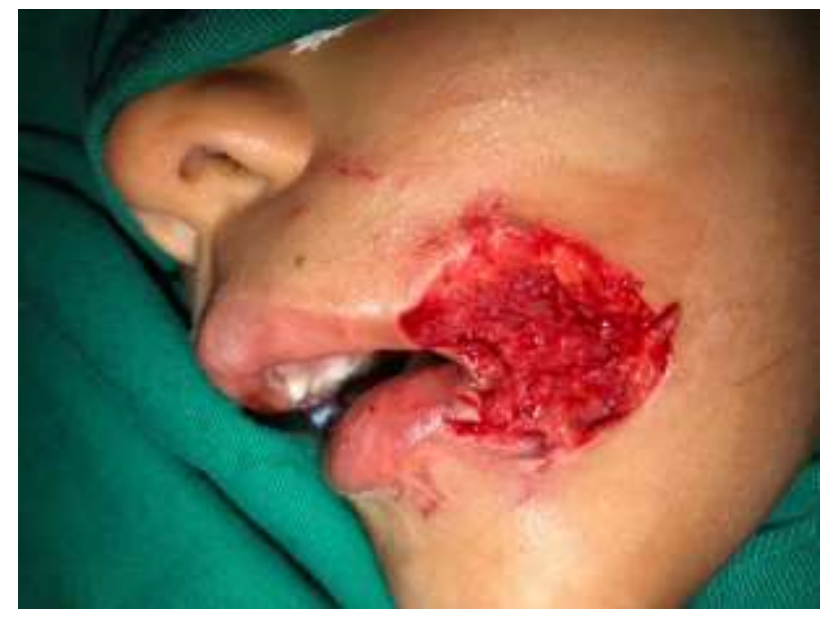

Fonte: Autores.

$\mathrm{O}$ tratamento instituído neste primeiro momento foi por meio de assepsia local da pele e ferimento com o uso de clorexidina $2 \%$ seguido de irrigação copiosa a base de solução salina, em seguida foi realizada a sutura em todo o ferimento e encaminhamento para vacinação anti-rábica após três dias. A figura 2 demonstra como ficou o resultado final da sutura.

Figura 2: Fotografia do término da sutura.

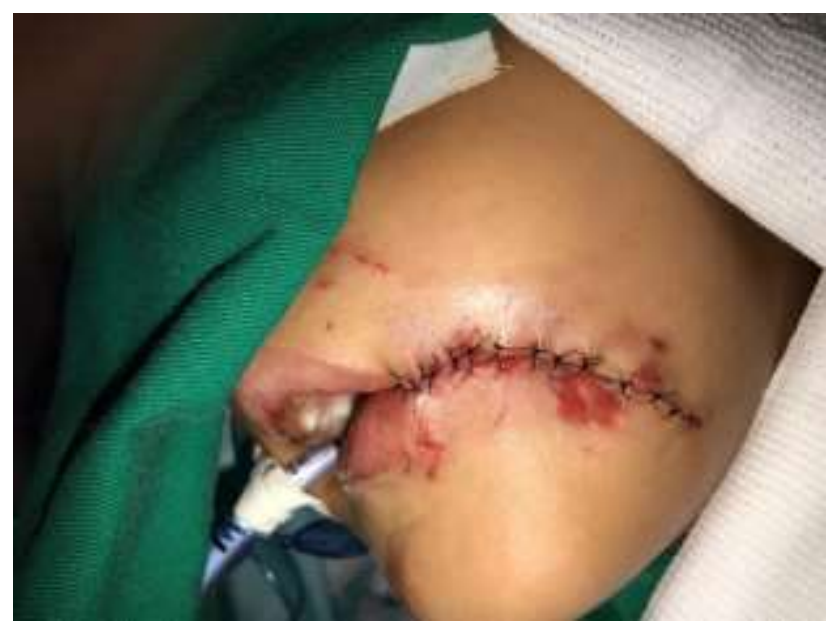

Fonte: Autores.

Foram dadas as orientações pós-operatórias de realização de curativos diários, retorno em 10 dias para remoção das suturas, retorno para acompanhamento da vacinação anti-rábica, repouso por 10 dias e orientação de higiene oral.

No acompanhamento do pós-operatório de 15 dias, o paciente apresentava cicatrização insatisfatória com um único ponto de necrose tecidual. Por causa disto, foram dadas as seguintes orientações pela equipe da estomaterapia: curativos diários com gazes úmidas com soro, mais óleo Dersani e também gel de Pielsana, não molhar em banho se trocar, e fazer isto a cada 12 horas até toda a necrose sair. Não foram realizados debridamentos mecânicos, o tratamento para esse foco de necrose foi apenas com protocolo medicamentoso. 
Figura 3: Fotografia do acompanhamento após 15 dias.

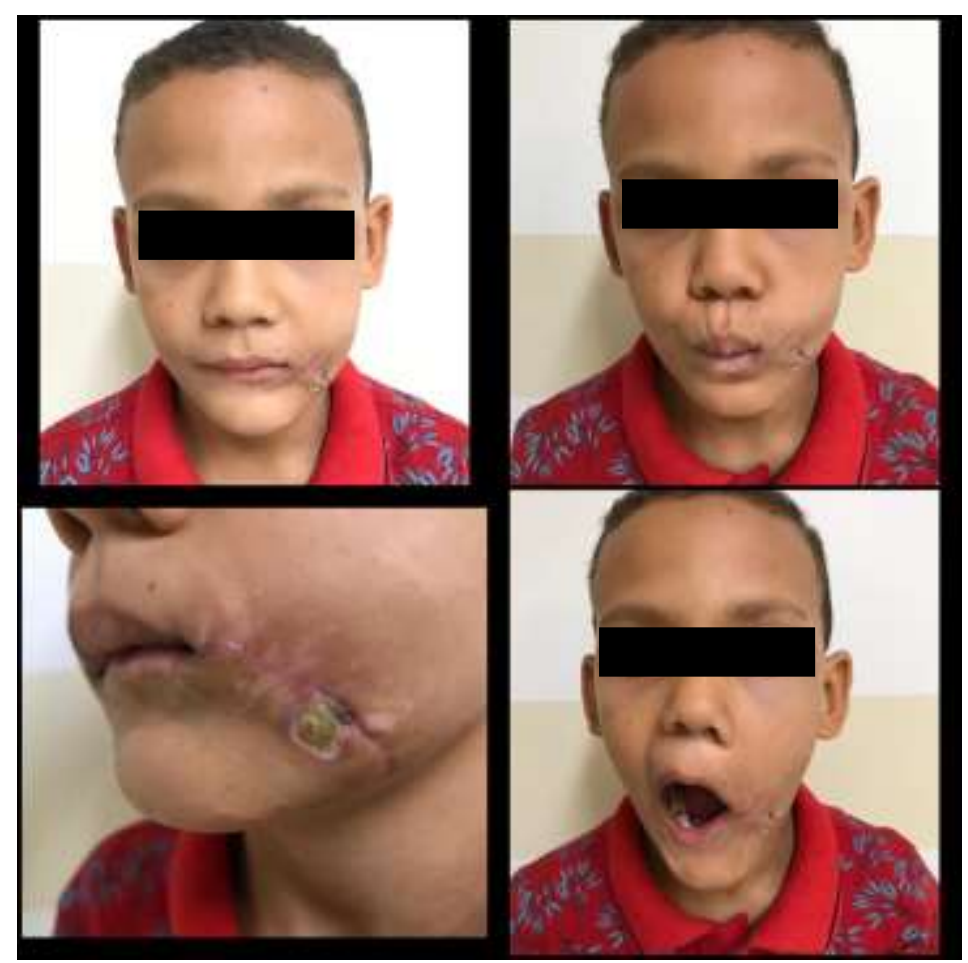

Fonte: Autores.

O óleo de Dersani foi indicado para auxiliar no processo de cicatrização destas feridas, pois promove um debridamento autolítico de feridas necróticas. O gel de Pielsana é para limpeza da ferida, sendo indicado para debridamento, hidratação e eliminação de odores.

Após 40 dias decorridos da lesão, o paciente retornou para consulta. Nesta consulta nota-se melhora substancial do ferimento, não havia mais focos de necrose e a pele encontrava-se bem cicatrizada. Não havia mais grandes limitações funcionais. Após isto, foi realizado encaminhamento para tratamento com fonoaudiólogo. A figura 4 demonstra o descrito. 
Figura 4: Fotografia de retorno após 40 dias.

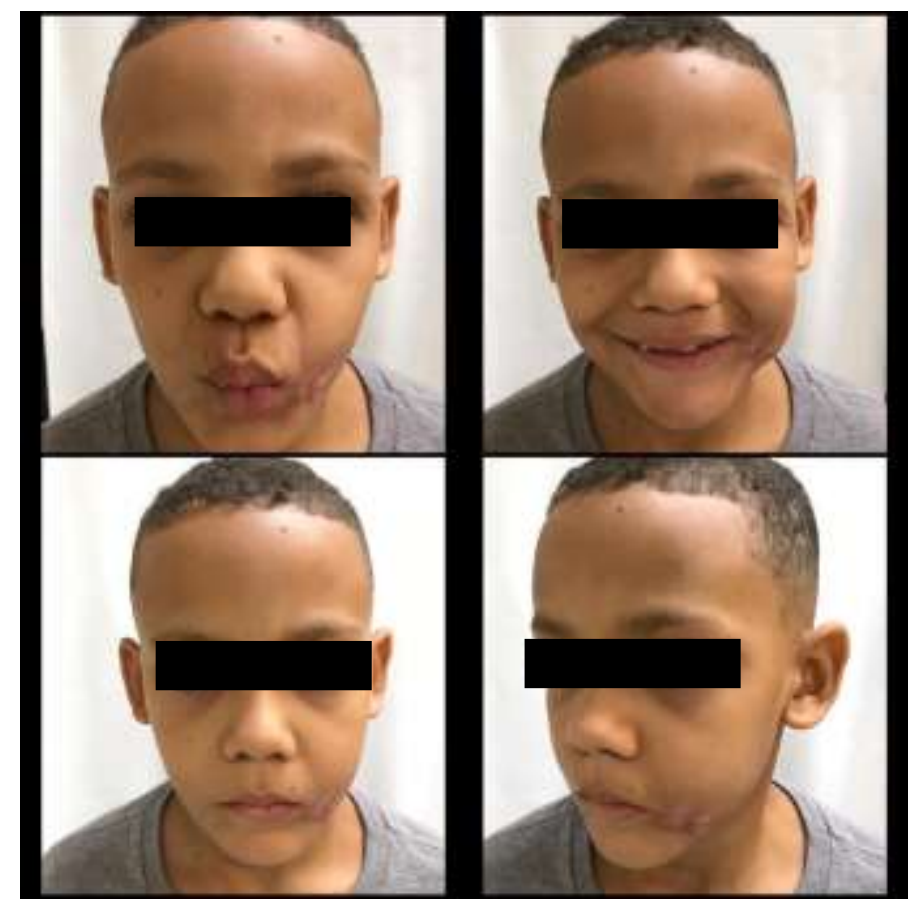

Fonte: Autores.

\section{Discussão}

O perfil de paciente acometido pelas lesões provindas de mordeduras caninas é em sua maioria de caráter pediátrico. Com uma faixa etária variável relatada na literatura, estudos apontam a maior prevalência desse tipo de lesão em pacientes com idade entre 8 a 14 anos, como relatado no presente caso, seguido de pacientes que apresentam entre 1 a 7 anos de idade (Carvalho \& Silva, 2007; Heitz et al., 2018; Saadi et al., 2018; Essig et al., 2018).

A ocorrência desse tipo de lesão em sua maioria apresenta predileção pelo sexo masculino em relação ao feminino, fator esse justificado pela diferença comportamental e questão cultural entre os gêneros, assim como no caso supracitado. (Carvalho \& Silva, 2007; Heitz et al., 2018)

As regiões mais acometidas por lesões de mordedura são, os membros inferiores e superiores, seguidos da região da cabeça e pescoço, e por fim tronco e demais regiões do corpo. O tipo de lesão encontrada em sua maioria é de aspecto profundo, particularmente no paciente pediátrico, esse fator é justificado pela espessura tecidual de caráter mais fino (Saadi $e t$ al., 2018; Eppley \& Schleich, 2013). No paciente pediátrico, a baixa estatura e a ausência de movimentos de defesa justificam o maior acometimento da região da cabeça e pescoço, destacando as áreas de lábio, nariz e bochechas, como relatado no presente caso (Piccart et al., 2018; Essig, Sheehan, Niermeyer, Lopez \& Elmaraghy, 2019; Eppley \& Schleich, 2013; Touré, Angoulangouli \& Méningaud, 2015).

A literatura aponta que traumas provindos de mordeduras caninas apresentam uma alta energia cinética que varia entre 50 e $100 \mathrm{~kg} / \mathrm{cm}^{2}$, no entanto, a ocorrência de fraturas associadas a esse tipo de lesão apresenta uma baixa taxa de prevalência (Heitz et al., 2018; Essig et al., 2018; Lackmann et al., 1992). Uma taxa de apenas 1 a 6\% dos casos reportados apresentam associação com a ocorrência de fraturas na região da cabeça, em traumas de mordeduras caninas na região facial de pacientes pediátricos (Saadi et al., 2018). 
Os fatores que justificam a baixa prevalência de fraturas faciais nesse tipo de lesão relacionam-se com a grande proporção de tecido ósseo esponjoso e de estruturas cartilaginosas no paciente pediátrico, fazendo com que assim haja uma maior absorção da energia do impacto (Heitz et al., 2018). No presente caso, o paciente não apresentou fraturas faciais ou comprometimento de estruturas ósseas provindos do trauma.

O tratamento inicial preconizado é dependente do nível e gravidade da lesão apresentada, podendo este ser desde um atendimento de emergência baseado no Advanced Trauma Life Support (ATLS) em casos mais severos, até um tratamento de nível ambulatorial em casos mais leves e moderados (Carvalho \& Silva, 2007; Heitz et al., 2018).

Inicialmente faz-se a limpeza copiosa com o uso de solução salina, seguido do desbridamento do tecido desvitalizado, fechamento primário das lacerações por meio de suturas, e estabelece-se então o tratamento de antibioticoterapia. Admite-se o uso de penicilinas como primeira escolha, sendo a amoxicilina associada ao ácido clavulonato como padrão ouro. O uso da eritromicina ou cefalosporinas de $1^{a}$ geração é admitido em casos de alergias provindas das penicilinas (Heitz et al., 2018; Saadi et al., 2018; Piccart et al., 2018; Lackmann, Draf, Isselstein \& Tollner, 1992; Cavalcanti et al., 2017). No entanto, no caso supracitado, admitiu-se o uso de cefalosporinas de $3^{\circ}$ geração como primeira escolha, por via endovenosa, onde se foi utilizado a Ceftriaxona EV na posologia de $1 \mathrm{~g}$ a cada 12 horas, associado também ao uso do Metronidazol EV na posologia de $500 \mathrm{mg}$ a cada 8 horas, pelo período de 5 dias. Após a alta hospitalar, instituiu-se o uso da Amoxicilina associada ao Ácido Clavulonato na posologia de $875 \mathrm{mg}+125 \mathrm{mg}$ pelo período de 7 dias, por via oral.

Outro fator importante que ronda o tratamento de mordeduras caninas é a necessidade da admissão do protocolo de vacinação antirrábica. A raiva é uma doença oriunda de uma infecção viral do sistema nervoso central provinda da contaminação do ferimento através da saliva do animal raivoso. No caso supracitado, o animal de origem não doméstica não apresentava dados comprobatórios de vacinação para se admitir como um animal protegido contra a raiva (Carvalho \& Silva, 2007; Heitz et al., 2018; Alencar et al., 2015). Portanto seguiu-se o protocolo de vacinação antirrábica instituído em 4 doses, sendo administradas nos seguintes dias: $0,3,7$ e 14.3

Apesar do protocolo instituído seguir a recomendação preconizada na literatura, o paciente evoluiu com área de necrose tecidual durante a fase de cicatrização. A necrose tecidual pode ser considerada como uma das complicações póstrauma por mordedura, assim como a cicatriz hipertrófica, fobia a cachorros, desordens do sono e choque psicológico (Touré et al., 2015).

Estudos apontam que o uso de substâncias tópicas como o óleo de girassol (óleo dersani) e substâncias compostas por ácidos graxos (gel pielsana), se mostram eficazes no estímulo à produção de tecido de granulação em casos de difícil cicatrização, fator esse determinado pela ação do ácido linoléico, que apresenta a capacidade de mediar processos bioquímicos que precedem a mitogênese de células fibroblásticas (Morais, Barros, Tamos \& Zuim, 2013). Assim como no relato supracitado, profissionais da estomaterapia, especializados na área de reparação de feridas, admitiram o uso do Óleo de Dersani e Gel de Pielsana como medida de tratamento para reparação e efetiva cicatrização da área necrótica.

\section{Conclusão}

O trauma promovido pela mordedura canina é definido como um problema de saúde público e que afeta em sua maioria a população pediátrica.

O manejo e acompanhamento desses pacientes é de extrema importância para uma melhor evolução do quadro clínico, direcionando dessa forma, a atenção da equipe multidisciplinar tanto no aspecto de prevenção a doenças de caráter mais severo como a raiva e tétano que provém de traumas dessa natureza, como também do processo de reparação tecidual. 
Observa-se um grande aparato de estudos que rondam esse tipo de trauma, e constata-se a eficácia do tratamento preconizado a base de assepsia e irrigação copiosa, fechamento primário da lesão tecidual, prescrição antibioticoterápica e acompanhamento do quadro de vacinação do paciente, assim como o acompanhamento efetivo pela equipe da estomaterapia na reparação tecidual.

\section{Referências}

Alencar, M. G. M. D., Bortoli, M. M. D., Almeida, H. C. R. D., Moraes, P. K. M. D., Lima, N. R. D., \& Vasconcelos, B. C. D. E. (2015). Reconstrução de lesão em lábio superior por mordedura animal em criança. Revista de Cirurgia e Traumatologia Buco-maxilo-facial, 15(4), 53-58.

Carvalho, C. C., \& da Silva, B. T. F. (2007). Características epidemiológicas de acidentes por mordedura de cão atendidos em unidade básica de saúde no nordeste do Brasil. Revista Brasileira em Promoção da Saúde, 20(1), 17-21.

Cavalcanti, A. L., Porto, E., Dos Santos, B. F., Cavalcanti, C. L., \& Cavalcanti, A. (2017). Facial dog bite injuries in children: A case report. International journal of surgery case reports, 41, 57-60.

Cohen-Manheim, I., Siman-Tov, M., Radomislensky, I., Peleg, K., \& Israel Trauma Group (2018). Epidemiology of hospitalizations due to dog bite injuries in Israel, 2009-2016. Injury, 49(12), 2167-2173.

De Klerk, P., Van Dijk, M., \& Van As, A. B. (2016). Treatment and outcome of unusual animal bite injuries in young children. South African medical journal = Suid-Afrikaanse tydskrif vir geneeskunde, 106(2), 206-209.

Drumright, B., Borg, B., Rozzelle, A., Donoghue, L., \& Shanti, C. (2020). Pediatric dog bite outcomes: infections and scars. Trauma surgery \& acute care open, 5(1), e000445.

Eppley, B. L., \& Schleich, A. R. (2013). Facial dog bite injuries in children: treatment and outcome assessment. The Journal of craniofacial surgery, 24(2), $384-386$.

Essig, G. F., Jr, Sheehan, C. C., Niermeyer, W. L., Lopez, J. J., \& Elmaraghy, C. A. (2019). Treatment of Facial Dog Bite Injuries in the Emergency Department Compared to the Operating Room. OTO open, 3(3), 2473974 X19858328.

Essig, G. F., Jr, Sheehan, C., Rikhi, S., Elmaraghy, C. A., \& Christophel, J. J. (2019). Dog bite injuries to the face: Is there risk with breed ownership? A systematic review with meta-analysis. International journal of pediatric otorhinolaryngology, 117, 182-188.

Foster, M. D., \& Hudson, J. W. (2015). Contemporary update on the treatment of dog bite: injuries to the oral and maxillofacial region. Journal of oral and maxillofacial surgery: official journal of the American Association of Oral and Maxillofacial Surgeons, 73(5), 935-942.

Heitz, C., Louzada, G. P., Conci, R. A., Rodrigues, R. L., \& Fritscher, G. G. (2018). Primary Repair of a Complex Panfacial Fracture by Dog Bite. Plastic and reconstructive surgery. Global open, 6(4), e1719.

Lackmann, G. M., Draf, W., Isselstein, G., \& Töllner, U. (1992). Surgical treatment of facial dog bite injuries in children. Journal of cranio-maxillo-facial surgery: official publication of the European Association for Cranio-Maxillo-Facial Surgery, 20(2), 81-86.

Macedo, J. L., Rosa, S. C., Queiroz, M. N., \& Gomes, T. G. (2016). Reconstruction of face and scalp after dog bites in children. Revista do Colegio Brasileiro de Cirurgioes, 43(6), 452-457.

Morais, D. C. M., Barros, P. O., Tamos, E. F., \& Zuim, N. R. B. (2013). Ação cicatrizante de substâncias ativas: d-pantenol, óleo de girassol, papaína, própolis e fator de crescimento de fibroblastos. FOCO: caderno de estudos e pesquisas, (4), 83-97.

Piccart, F., Dormaar, J. T., Coropciuc, R., Schoenaers, J., Bila, M., \& Politis, C. (2019). Dog Bite Injuries in the Head and Neck Region: A 20-Year Review. Craniomaxillofacial trauma \& reconstruction, 12(3), 199-204.

Saadi, R., Oberman, B. S., \& Lighthall, J. G. (2018). Dog-Bite-Related Craniofacial Fractures among Pediatric Patients: A Case Series and Review of Literature. Craniomaxillofacial trauma \& reconstruction, 11(4), 249-255

Senturk, E., Dagistanli, N., Calim, O. F., \& Ozturan, O. (2019). Nasal Reconstruction Following a Dog Bite. The Journal of craniofacial surgery, 30(7), 22332235 .

Touré, G., Angoulangouli, G., \& Méningaud, J. P. (2015). Epidemiology and classification of dog bite injuries to the face: A prospective study of 108 patients. Journal of plastic, reconstructive \& aesthetic surgery: JPRAS, 68(5), 654-658.

Vieira, C. L., Araújo, D. C. D. C., Ribeiro, M. L. S., \& Laureano Filho, J. R. (2013). Lesão de tecido mole em pacientes vítimas de trauma buco-maxilofacial. Revista de Cirurgia e Traumatologia Buco-maxilo-facial, 13(1), 97-104.

Zhu, N., Cruz Walma, A., Troulis, M. J., \& August, M. (2020). Facial dog bites treated at the Massachusetts General Hospital over a 20 -year period. Oral surgery, oral medicine, oral pathology and oral radiology, 130(2), 136-143. 